\title{
Verbal interference with visual classification: Optimal processing and experimental design
}

\author{
JOHN H. FLOWERS and BECCY BLAIR \\ University of Nebraska-Lincoln, Lincoln, Nebraska 68588
}

\begin{abstract}
The speed of classification of six alternative ink colors into two categories of three colors each was measured in tasks for which the colors were displayed as either XXXX patterns or incongruent words (Stroop stimuli). Substantial interference from the words occurred when the classification required grouping nonadjacent hues (red, yellow, and blue vs. orange, green, and purple) regardless of whether subjects received exclusive practice with this classification. Interference also occurred when the classification required grouping adjacent hues (red, orange, and yellow vs. green, blue, and purple), but only if this classification was intermixed within blocks of trials with the nonadjacent classification; subjects who received exclusive practice with the adjacent classification sorted the Stroop stimuli as rapidly as the XXXX stimuli.
\end{abstract}

Performance speed as assessed by choice reaction time or speeded classification techniques provides one measure of the efficiency with which human observers can gate or filter irrelevant stimulus information in a display (Garner, 1974; Posner, 1964). Since selective attention research is often concerned with optimal processing by reasonably practiced human observers (e.g., Friedman \& Derks, 1973), the selection of an experimental design should provide an adequate opportunity for observers to adjust to task demands and strategies appropriate to individual conditions. When an experiment involves several conditions requiring different optimal processing strategies which may not be immediately obvious to the observer (Felfoldy \& Garner, 1971), different methods of ordering stimulus conditions might conceivably lead to different conclusions about the efficiency of selective attention.

Flowers and Dutch (1976) measured the speed of visual scanning for sets of one to three color patches which were displayed as either XXXXs or as incongruent color names (Stroop stimuli). Substantially slower scanning rates were observed for the Stroop stimuli than for the XXXX stimuli when the task required searching for three widely distributed hues (e.g., orange, green, and purple). However, when subjects had to scan for only a single color, or a grouping of two or three similar colors (e.g., green, blue, and purple), no verbal interference effect was noted. These results suggest the use of an efficient visual stimulus code which allows rapid stimulus classification independently of verbal processing, provided the visual memory load is sufficiently small.

This research was supported by a research graint-in-aid from the University of Nebraska Research Council, and was conducted while the first author was supported by a Senior Faculty Summer Research Fellowship provided by the University of Nebraska Research Council.
Furthermore, this memory load is not determined by set size alone, but also by the sensory heterogeneity of the stimuli within the set.

In a speeded classification task requiring the sorting of six different colors into two categories of three colors each, Flowers and Dutch found a verbal interference effect even when the colors within response categories were adjacent hues (e.g., red, orange, and yellow vs. green, blue, and purple), suggesting that visual classification of colors independently of verbal coding may be less efficient with sorting than with scanning. However, in their card sorting task, Flowers and Dutch intermixed trials requiring the red, orange, and yellow vs. green, blue, and purple split (ROY/GBP) with trials requiring a red, yellow, and blue vs. orange, green, and purple split (RYB/OGP). It seems possible that the inclusion of the RYB/OGP classification might have precluded the learning of an optimal visual (nonverbal) processing strategy with the ROY/GBP task; for example, verbal rehearsal might have been required by the larger memory load in the RYB/OGP task, while verbal rehearsal would have been counterproductive in the ROY/GBP task. In order to investigate this possibility, and also provide an additional assessment of the ability of subjects to avoid Stroop interference in classification tasks of this type, the present experiment was conducted.

\section{METHOD}

\footnotetext{
Subjects

Twenty-four volunteers from an introductory psychology course each served in a single experimental session lasting about $40 \mathrm{~min}$ to $1.5 \mathrm{~h}$, depending upon the conditions assigned. All subjects had normal color vision and normal or corrected acuity.

\section{Tasks}

Each experimental trial required the sorting of a deck of 30 $8.9 \times 6.3 \mathrm{~cm}$ white stimulus cards into two piles of 15 cards
} 
each, according to the hue of the color patch attached to each card. Two different decks of cards were used. The $X$ deck contained five instances each of cards containing red, orange, yellow, green, blue, and purple color patches; each color patch was displayed as XXXX in boldface capital letters about $.5 \mathrm{~cm}$ high mounted in the upper third of each card. The $W$ deck contained five instances each of each hue, but the color patches were displayed as incongruent color names (the color names were equally distributed among the other hues contained in the deck). Two different color classifications were required. ROY/GBP required a red, orange, and yellow vs. a green, blue, and purple split, while RYB/OGP required a red, yellow, and blue vs. orange, green, and purple split. The combination of the two decks and the two color classifications thus produced a total of four different sorting tasks.

\section{Procedure}

Before beginning the experiment, each subject was seated at a table and shown examples of the cards. He/she was then instructed that all tasks would require sorting the cards on the basis of the ink color of the stimuli only and not on the configuration or spelling of the ink patches. Prior to beginning each trial, the stimulus deck was shuffled and a card containing examples of the ink colors which were to be sorted into each category was shown to the subject. This card was removed from view when the subject indicated that he/she understood the required classification. On each trial, subjects held the deck in one hand, and on the oral signal of "Ready, set, go!" dealt each card in to the required pile "as rapidly as possible, avoiding errors." Sorting times were measured with a stopwatch; both time and errors were recorded following each trial. Subjects were informed about errors but not about times.

\section{Presentation Order: Within-Subjects vs. Between-}

Subjects Designs

One group of eight subjects received eight experimental trials in tasks ROY/GBP-X and ROY/GBP-W only. These two conditions were given alternately on 16 successive trials, with four of the subjects beginning with ROY/GBP-X and the remaining subjects beginning with ROY/GBP-W. A second group of eight

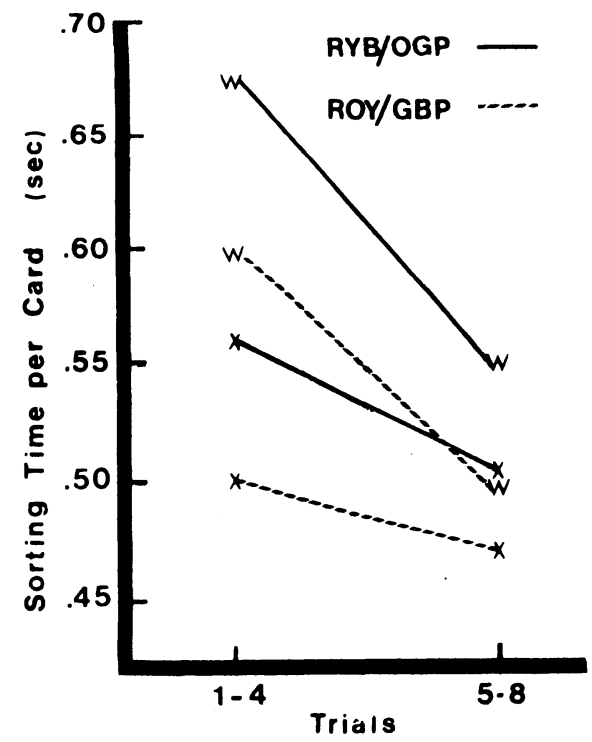

Figure 1. Mean sorting times per card for the $X$ decks and $W$ decks in the two color classification tasks. Data are from subjects who received all four deck and task combinations (within subjects design).

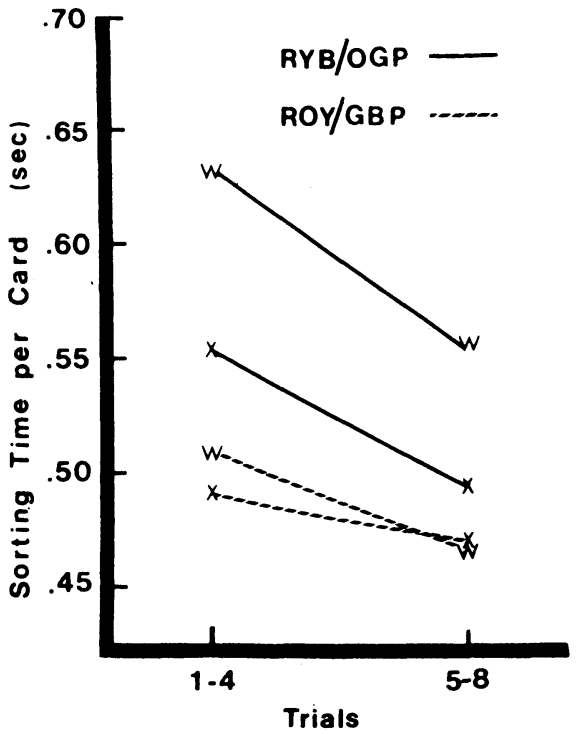

Figure 2. Mean sorting times per card for the $X$ decks and $W$ decks in the two color classification tasks. Independent groups of subjects were used for the RYB/OGP and ROY/OGP tasks (between subjects design).

subjects received on alternate trials RYB/OGP-X and RYB/OGP.W, also providing a total of eight sortings of the two tasks. These two groups of subjects thus served in an experiment in which the color classification was a between-subjects variable.

A third group of eight subjects received all four tasks; the order of presentation was block randomized in eight blocks of trials; each task thus occurred once in each block. This third group of subjects thus served in an experiment in which the color classification was a within-subjects variable, as was the case in Flowers and Dutch (1976).

\section{RESULTS}

Figures 1 and 2 display the mean sorting time per stimulus averaged across the first four and the last four blocks of trials for each combination of color classification and stimulus type. The data in Figure 1 is from the eight subjects who received all four conditions (the within-subjects experiment), while Figure 2 displays data from subjects who received only one color classification (between-subjects experiment). As both figures suggest, there was a substantial difference between the classification times for RYB/OGP-X and RYB/OGP-W in both experiments $[F(1,7)=15.8$ and 22.7 , respectively; $\mathrm{p}<.01$ in each case]. A slight decrease in the magnitude of this interference effect over trials is evident from the Deck Type by Blocks interaction $[F(1,7)=9.7, p<.05$ for the within subjects group, and $F(1,7)=4.2, p<.10$ for the between subjects group], but this appears largely to result from differential room for improvement with practice. Thus, substantial interference from incongruent 
words in a task requiring the classification of colors into dissimilar groupings appears to occur even when subjects are given practice exclusively on one color classification.

With the within-subjects design, a somewhat smaller, but nevertheless significant, verbal interference effect occurred with the ROY/GBP classification $[F(1.7)=8.2, p<.05]$, and while there is again a small interaction with practice $[F(1,7)=6.1, p<.05]$ there is still a significant difference between the mean sorting times for the $\mathrm{X}$ and $W$ decks during the last four trials $[t(7)=2.51$. $\mathrm{p}<.05]$. This pattern of data is therefore nearly identical to that obtained by Flowers and Dutch (1976).

On the other hand, the data from the between-subjects experiment reveals no significant differences between the sorting times for ROY/GBP-X and ROY/GBP-W (F $<1)$. Even in the first four trials, the 20 -msec difference between the means does not meet statistical significance $[\mathrm{t}(7)=1.35]$. It is thus apparent that unlike the subjects in either the within-subjects group in the present study, or in the previous experiment by Flowers and Dutch (1976), those subjects who received only the $\mathrm{ROY} / \mathrm{GBP}$ classification were able to perform without susceptibility to verbal interference.

\section{DISCUSSION}

Together with the findings of Flowers and Dutch (1976), the present results clearly illustrate the importance of the visual encodability of a response grouping of colors in determining whether speeded classification can occur independently of verbal processing. There would indeed appear to be an information limit within which visual attributes necessary to discriminate groupings of colors can be efficiently stored and rapidly compared with visual input at a sensory rather than verbal level. When a task requires the storage of more than this amount of information (as is apparently the case when subjects must group red, blue, and yellow into the same category), verbal processes which are subject to Stroop-like interference appear to become operative.

Additionally, however, the present findings provide an illustration of how the ability of subjects to perform optimally in a task requiring selective attention between relevant visual and irrelevant verbal attributes is quite dependent upon the particular experimental design chosen-specifically whether subjects are given massed practice with a single task, or whether tasks are distributed within blocks. It seems possible that the necessity of a verbal encoding or rehearsal strategy 1 for performing the RYB/OGP classification may have led to the use of a counterproductive encoding strategy with the ROY/GBP classification. Regardless of the precise "locus" of the verbal interference, the present results point to the potential hazard of exclusively using within-subjects designs to make generalizations about optimal human information processing.

\section{REFERENCES}

Felfoldy, G. L., \& Garner, W. R. The effects on speeded classification of implicit and explicit instructions. Perception \& Psychophysics, 1971, 9, 289-292.

Flowers, J. H., \& Dutch, S. The use of visual and name codes in scanning and classifying colors. Memory \& Cognition, 1976, in press.

Friedman, H., \& Derks, P. L. Simultaneous motor and verbal processing of visual information in a modified Stroop test. Perception \& Psychophysics, 1973, 13, 113-115.

GARNER, W. R. Attention: The processing of multiple sources of information. In E. C. Carterette \& M. P. Friedman (Eds.), Handbook of Perception (Vol. 2). New York: Academic Press, 1974.

PoSNER, M. I. Information reduction in the analysis of sequential tasks. Psychological Review, 1964, 71, 491-504.

\section{NOTE}

1. Subjects in this experiment and in previous experiments (Flowers \& Dutch, 1976) have informally reported using verbal rehearsal to aid in the classification of nonadjacent hues.

(Received for publication November 10, 1975.) 